\title{
THE CASSEL MEETING OF THE DEUTSCHE MATHEMATIKER-VEREINIGUNG.
}

THe annual meeting of the Deutsche Mathematiker-Vereinigung was held, in connection with the seventy-fifth convention of the Deutsche Naturforscher und Aerzte, at Cassel, September 20-26, 1903. More than two thousand persons attended the convention and were royally entertained by the citizens of Cassel. A reception to visitors, on Sunday evening, marked the formal opening of the convention; on Monday and Friday mornings occurred general meetings in the Stadtpark; and on Thursday morning the regular business meeting was held. The other mornings and afternoons of the week were devoted to the reading of papers before the respective sections, which met in different parts of the city.

The weather was ideal and many excursions were arranged to different parts of the surrounding country, in particular to the wonderful artificial waterfall and to the Emperor's summer castle on Wilhelmshöhe, about five miles west of the city. Visits were also made to the extensive locomotive works situated here. Cassel has a fine picture gallery which was well patronized during the week. Each evening had its own particular entertainment, beginning Monday evening with an opera, seats to which were reserved for all registered visitors. Tuesday evening occurred a banquet and on Friday evening a "Trinkfest" at the expense of the city of Cassel. On Wednesday evening the members of the Mathematical Section arranged to dine together.

An invitation was extended by the faculty and officers of the University of Göttingen to visit the university Friday afternoon and Saturday morning for the purpose of inspecting the equipment, particularly in the departments of mathematics, chemistry, physics, astronomy, and agriculture. About one hundred and thirty persons accepted the invitation.

A committee appointed to consider the question of founding a central mathematical library reported that the firm of B. G. Teubner, in Leipzig, had offered to present a complete set of its publications as a nucleus for the library and that the firm further offered to assist in cataloguing its gift. The offer was gratefully accepted. 
The next meeting of the Vereinigung will be held at Heidelberg, August 8-13, 1904, in connection with the third International mathematical congress. Notices of this congress have already been published in the BuLlETIN (volume 9, page 565 ; volume 10 , page 94 ).

At the Cassel meeting the Vereinigung under the chairmanship of Professor Klein held six sessions, at which the following papers were read :

1. Professor Scheffers, Darmstadt: "Sophus Lie."

2. Professor LaMPe, Berlin : "On the life and work of M. Hamburger."

3. Professor Fricke, Braunschweig: "On new methods and new text-books in England."

4. Dr. Geissler, Charlottenburg: "Foundations of noneuclidean geometry subject to conditions on the infinite elements."

5. Dr. Bernstein, Halle : "On abelian 'Körper' in an imaginary fundamental domain."

6. Dr. Blumenthal, Göttingen: "On abelian functions and modular functions of several variables."

7. Professor Boltzmann, Vienna : "On an extension of the Lagrangean equations for the case of non-holonome coördinates."

8. Professor Burkhardt, Zürich : "Report on the development of the oscillatory functions."

9. Dr. Hamel, Carlsruhe: "On the meaning of the Lagrangean 'Transitivitätsgleichungen' in mechanics."

10. Professor Heffeter, Bonn: "On systems of teaching geometry, particularly in case of analytic treatment."

11. Dr. Liebma nN, Leipzig: "A general proof of the Minding theorem on the stability of closed convex surfaces."

12. Professor Maschke, Chicago : "On invariants of quadratic differential forms."

13. Professor Menmke, Stuttgart : "On graphic kinematics and dynamics."

14. Professor Meyer, Koenigsberg: "On a fundamental equation of surface theory and mechanics."

15. Professor Schoute, Groningen : "Considerations on the content of an $n$-dimensional prismoid."

16. Professor StÄCKEL, Kiel : "Report on the mechanics of multiple manifolds."

17. Professor Schoenflies, Koenigsberg: "On analysis situs." 
18. Professor JueL, Copenhagen : "Volumes of pyramids."

19. Professor Wien, Würzburg: "On differential equations of electrodynamics of moving bodies."

20. Professor Hilbert, Göttingen : "Mechanics of continua."

21. Professor Mrnkowski, Göttingen : "On capillarity."

22. Dr. Wellstern, Giessen: "Fundamental deductions from the arithmetic theory of algebraic functions of a single variable."

23. Professor Wiener, Darmstadt: "The problem of the normal to a conic."'

24. Professor Prandte, Hanover: "On uniform symbolism in vector analysis."

25. Professor Wiener, Darmstadt: "Exhibition of mathematical models."

Professor Maschke's paper has already appeared in the Transactions of the American Mathematical Society. The first two papers of the programme were biographical rather than mathematical; no abstracts of these are offered. Abstracts of the other papers, so far as obtainable, are as follows :

3. Professor Fricke outlined what Professor Perry is endeavoring to do in England and compared the Perry movement in England with a parallel movement in the German universities. He also exhibited copies of English text-books of elementary mathematics. An extended report on the subject will appear in the Jahresbericht of the Vereinigung.

4. Dr. Geissler extended the archimedean continuity of the finite to a general continuity of the infinite. This theory is not in part discontinuous but is in general continuous. Similarily, the hypereuclidean geometry which he is about to publish is not anti-euclidean or non-euclidean, but is in the same domain as euclidean geometry. Among the new concepts in this book are the following: There exist several parallels through one point which cut a second straight line in different points at infinity; the theorem on the sum of angles is, in many cases, independent of the axiom of parallels.

5. Dr. Bernstein bases his work on theorems of Kronecker, Weber, and Hilbert. He gives the necessary and sufficient conditions that a "Primideal" of the fundamental system (Grundkörper) shall lie in a particular class of the same. He gives further, on the ground of empirical induction, an exact 
criterion in the case of a "Primideal" of the fundamental system in the corresponding "Klassenkörper."

6. Dr. Blumenthal showed that as the Jacobian modular function may be represented as the quotient of null values of $\vartheta$-functions of a single variable, so the modular function of several variables (see Mathematische Annalen, volume 56) may be expressed by means of quotients of null values of $\vartheta$-functions of several variables. This theorem, which originated with Professor Hilbert, rests on the theory of algebraic "Zahlkörper" and can be applied for any arbitrary number of variables. The paper will appear in the Jahresbericht.

7. Professor Boltzmann showed how the correction terms which the Lagrangean equations require are to be computed in case the generalized coördinates $p$ which are used are not holonome, $i$. e., where a non-integrable relation of the form

$$
d x=\sum_{h} d p_{h} f_{h}\left(p_{1}, p_{2}, \cdots, p_{n}\right)
$$

exists between the coördinates $p$ and the rectangular coördinates $x$. He proposes, further, whenever the law according to which the forces act, as well as the determining equations, involves the time explicitly, that the given mechanical system shall be called a "skleronom" system. Similarly the generalized coördinates shall be called skleronom if they are connected with the rectangular coördinates by equations which do not contain the time explicitly. The opposite case he calls " rheonom."

8. Professor Burkhardt has collected and arranged in chronological order material on the development of oscillatory functions. Already three parts, comprising 768 pages, have appeared in volume 10 of the "Jahresbericht," in the years 1901, 1902, 1903, respectively. The fourth and last part is now in press. Professor Burkhardt's paper was a brief report on the contents of this fourth part, emphasizing some of the more recent and important developments in the subject.

9. Dr. Hamel proposed the problem : A generalization of the Lagrangean equations of mechanics when the position of the system is represented by the coördinates $q_{1}, \cdots, q_{n}$, and the 
velocity of the system is represented, not by the derivatives $q_{1}^{\prime}, \ldots, q_{n}^{\prime}$ with respect to time, but by linear, otherwise arbitrary (hence non-holonom), relations

$$
\omega_{\lambda}=\sum_{\lambda=1}^{n} \pi_{\lambda} \rho q_{\rho}^{\prime} \cdots
$$

between these derivatives. In order to solve the problem it is necessary to deduce the "Transitivitätsgleichungen" which determine the variations $\delta \omega_{\lambda}$ in terms of the derivatives of the actual displacements

$$
\delta D_{\lambda}=\sum_{\lambda=1}^{n} \pi_{\lambda} \rho \delta q_{\rho} \cdots
$$

corresponding to equation (1).

Dr. Hamel illustrates the advantage of the Lagrange-Euler equations of mechanics in handling non-holonom equations of condition, and indicates briefly their relation to Lie's theory of transformation groups. His paper will appear in full in the Zeitschrift für Mathematik und Physik.

10. Professor Heffter's paper is in abstract as follows: If " point," " plane," and "straight line" are taken as elements of space, and " incidence," "parallelism," and "orthogonality" as the three elementary relations, then geometry separates into the three divisions : projective, affine, conform, corresponding to the three similarly named transformation groups. The projective geometry depends on incidence ; the affine on incidence and parallelism; the conform on incidence, parallelism, and orthogonality. In the analytic treatment the notion of a characteristic absolute invariant of transformation groups or geometry will be taken as fundamental. As an example, in plane projective geometry we have the anharmonic ratio of point or line pairs; for the affine geometry, the distance ratio of a straight line from point pairs; for conform geometry, the distance ratio of a point with respect to straight line pairs. The natural coördinates for each division of geometry will be chosen with the help of a particular characteristic absolute invariant.

11. Dr. Liebmann corrected an error in a previous paper and showed that the general spherical and closed convex surface 
is non-deformable only under initial assumptions on the nature of the deformation.

13. Professor Mehmke utilizes the hodograph (MöbiusHamilton) and shows how a graphic representation for $n$ dimensional space may be developed. For motion in space of more than three dimensions, a generalization of descriptive geometry is needed, which can be found in Professor Schoute's book on Mehrdimensionale Geometrie. As the acceleration is found from the velocity, so the "hyper-acceleration" (acceleration of $2 \mathrm{~d}$ order or velocity of $3 \mathrm{~d}$ order) can be found from the ordinary velocity and acceleration. In general the velocity of $(n+1)$ th order can be found from those of the first and $n$th orders.

The construction furnishes a general graphic solution of the problem : To determine the path of a point when, instead of the initial position and initial velocity, the accelerating force for each point is given, a problem which has been solved only for motion in a plane under particular restrictions. The paper will appear in full in the Zeitschrift für Mathematik und Physik.

14. The well known transformations that Lagrange has employed on his equations in dynamics, in which, according to assumptions in case of a function of forces, a material point is constrained to lie on a given surface, is from the standpoint of surface theory nothing other than a systematic introduction of Gauss's first surface form in the equation of motion. The question of a corresponding relation in the case of Gauss's second surface form has led Professor Meyer, without any restriction in respect to the force, to a simple law existing between the Huyghen's normal force (centrifugal or centripetal) belonging to the path curve and the tangential force belonging to the surface. The deduction for the radius of curvature of a curve on the surface is, in this case, extremely simple. An application of the law gives a general mechanical definition of geodesic lines as well as of asymptotic lines of a surface.

Different forms of the law are derivable by direct application of simple principles of mechanics and geometry. The law is also applicable to $n$-dimensional space.

15. Professor Schoute deduces new expressions for the volume of an $n$-dimensional prismoid, e. $g$., for $n=5$, 


$$
I=\frac{h}{18}\left[8 D_{\frac{1}{2}}+5\left(D_{\frac{1}{2}-\frac{1}{10} \sqrt{5}}+D_{\frac{1}{2}+\frac{1}{15} \sqrt{5}}\right)\right],
$$

and incidentally finds the following theorem :

If $1,2, \cdots, n-1, n$ be the roots of the equation

$$
a_{0} x^{n}+a_{1} x^{n-1}+\cdots+a_{n-1} x+a_{n}=0,
$$

the equation

$$
\frac{a_{0}}{n+2} x^{n}+\frac{a_{1}}{n+1} x^{n-1}+\cdots+\frac{a_{n-1}}{3} x+\frac{a_{n}}{2}=0
$$

admits only one pair of real roots for even $n$, and only one real root for odd $n$; the roots of the pair are equal to $n$, and the unique root lies between $n$ and $n+1$.

As Professor Schoute states, it is not this theorem itself, but its hyperdimensional origin that perhaps may be of interest.

16. Professor Stäckel reviews the notion of multiple manifolds of mechanies and takes up the following problems: The case of motion of a point in a multiple manifold in which the differential equations of the motion can be integrated by quadrature; the problem of the planets in non-euclidean space; finally the analytic equivalence of problems of dynamics and the application of Lie's theory of transformation groups to dynamics. He considers the relation between multiple manifolds of mechanics and numerous chapters of higher analysis and geometry on the one side and certain realms of mathematical physics on the other. In the latter connection the investigations of Gibbs are particularly considered.

17. The paradoxes of the "Mengenlehre" having completely upset the notions ordinarily connected with the words "curve," "surface," etc., it is the problem of the theory of "Punktmengen" to establish, by suitable definition, those conceptions with which analysis situs operates. These definitions are, moreover, to be such that they shall remain invariant under all "oneto-one" continuous transformations of the plane; for under these transformations all the theorems of analysis situs remain invariant. Professor Schoenflies shows how this can be easily established in case of the ordinary invariants of the group, namely, the dimension and relation in the realm of Punktmeng- 
en. The classification of all plane closely connected Punktmengen shows that the simple curve segment and the simple closed curve is the simplest type ; from these the "one-to-one" continuous representation on the circle segment and the circle is established. This method is applicable in space and here also leads to the theorems that are used in analysis situs, and of these the first is the theorem on curves by Jordan.

18. Professor Juel showed, by diagram, how a special foursided pyramid can be cut up and the parts again put together in the form of a cube. He called attention to a note by himself on this problem published in Berichten der Kgl. Gesellschaft der Wissenschaften zu Kopenhagen, 1903, under the title "Egalité par addition de quelques polyèdres." This same problem has been handled by Mr. Hill in the Proceedings of the London Mathematical Society, volume 27, but in a slightly different way.

19. Of the differential equations for bodies in motion the system of Lorenz is the only one that has stood the test throughout. These equations have been integrated with exactness only for the case of point charges moving with constant velocity. In Professor Wien's paper the integration has been completed for the case where, instead of stationary charges under known conditions, the generalized case of motion with constant velocity occurs. If for example

$$
F \frac{(c t-l)}{r}, \quad r^{2}=x^{2}+y^{2}+z^{2}
$$

is the general integral for rest, when $F$ is any arbitrary function, $c$ the velocity of light, $r$ the distance from a point $O$, then the integral for motion is

where

$$
F\left(\frac{c k t-v x / k-r}{r}\right),
$$

$$
r^{2}=x^{2} / k^{2}+y^{2}+z^{2}, \quad k^{2}=1-v^{2} / c^{2}
$$

One can use this to derive the dependence of the radiation of an electromagnetic vibration on the motion itself. For stationary vibrations the theory has been given by Herz. For 
the variable vibration an increase of the radiating energy occurs because of the motion, and in fact, is greater in case of transversal than of longitudinal vibration. For the velocity of light the radiating energy in unit time is infinite.

In case of a transversal vibration the system is such that for infinitely slow vibrations it does not give the same field as a moving electrified double point, according to the general theory. On the contrary there exists a field containing lines of force that vanishes with the vibrations, not with the radiations.

21. A report of Professor Minkowski's paper was to appear in Nature.

23. The problem of drawing the four normals to the conic $k$ from any point $n$. which is chosen at random in the plane of its conic was solved by Apollonius. Professor Wiener has treated this problem from the standpoint of modern mathematics, in particular using the theory of binary and ternary forms and has found a purely binary solution.

If we consider the point $n$ as the center of a point involution on the plane $k$ and desire to find, from the double points of this involution, the mixed polar system of the fourth order, the required polars are given by an involution of point pairs, or more exactly, by a set of polar systems of second order; and of these polar systems of the set we find the common apolar system of second order. The mixed polars of the fundamental points of this polar system to the binary polar system of order six is that polar system of fourth order whose fundamental points form the group of the required points. The solution depends then not really on the solution of an equation of the fourth degree but upon the determination of the polar system of the fourth order.

24. Professor Prandtl discusses the methods of representing vectors. He concludes that it is impracticable to use one alphabet (German) for vector quantities and another alphabet (Latin) for absolute values. He proposes that in script underscoring be employed, and in printing, heavy type. The operation symbols $=,+,-$, can then be employed as in algebra. For multiplication with a scalar the ordinary method of juxtaposition can be employed, as $u=g t$. The product of two vectors may be represented in accordance with Gibbs as follows: $a \cdot b$ means the direct product; $a \times b$ the skew product. 
If for pedagogical reasons a particular representation of an operation is desired, a double underscoring, or in case of multiplication parentheses can be employed.

25. In his second paper Professor Wiener explained the construction of new models of surfaces of the second order. Two thread models, the hyperboloid of revolution and the hyperbolic paraboloid, are so constructed that their forms may be changed without changing the length of the threads. Models of the same surfaces are made of light rods fastened together by a ball and socket device. Each rod is joined to other rods at three different points. The forms of these models can be changed. In the case of the hyperboloid of one sheet, the limiting forms, the ellipse and the hyperbola, are exhibited in an extremely neat form.

I wish to thank those who have so generously aided me, by by the loan of papers and otherwise, in the preparation of this report.

GötTINGen,

R. E. WILsON.

November, 1903 .

\section{ON A TEST FOR NON-UNIFORM CONVERGENCE.}

BY DR. W. H. YOUNG.

(Read before the American Mathematical Society, August 31, 1903.)

1. A PAPER of Cayley's entitled "Note on Uniform Convergence," appeared in 1893 in the Proceedings of the Royal Society of Edinburgh. It was reprinted in volume 13 (1897) of Cayley's Collected Works. The only reference to the paper which to my knowledge exists is a remark by Pringsheim in his article in Encyclopädie II A 1, page 34, to the effect that the objections made by Cayley in the paper in question to the usual definition of non-uniform convergence appear to be due to a misconception.

It is clear indeed from the discussion of the definition with which the note commences that Cayley had remained in what may be supposed to have been Stokes's order of ideas at the time of writing his* classical memoir on the subject in 1847,

* Stokes, Cambr. Math. Soc. Trans., vol. 7, p. 533 (1847). 